\title{
ANALYSIS ITEMS TO ASSESS THE QUALITY OF OPEN ONLINE COURSES FOR HIGHER EDUCATION
}

\author{
Marina Marchisio ${ }^{1}$ and Matteo Sacchet ${ }^{2}$ \\ ${ }^{1}$ Department of Molecular Biotechnology and Health Sciences, University of Torino, Via Nizza, 52, 10126, Torino, Italy \\ ${ }^{2}$ Department of Mathematics “G. Peano”, University of Torino, Via Carlo Alberto, 10, 10123 Torino, Italy
}

\begin{abstract}
The quality of Massive Open Online Courses (MOOC) is an important topic to be addressed by different stakeholders: higher education institutions, MOOC providers, education companies, educational providers. National and international agencies are or will be deeply involved in preparing or attending common guidelines in order to fulfill quality of MOOCs. Some indicators that can help in analyzing quality are provided by the learner point of view, by pedagogy, by instructional design, by outcome measures. The latter has been the most widely adopted, since numerical data help in comparison between different platform or different educational experiences. But these data, such as completion and retention rates, were criticized when used to assess quality of MOOCs. In the literature, there are some checklists and framework that can help and guide the expert or novice designer in the process of MOOC developments. In the present work, we are going to address the experience of the start@ unito project, an online platform developed at the University of Torino which offers 50 open online courses in order to facilitate the transition between secondary and tertiary education, making the students anticipate their career by attending a complete university module online prior to enrolment at the university. This could lead to an improvement in the number of ECTS credits acquired during the first year of university studies and to the drop-out rate. An analysis of the start@unito open online courses quality is provided according to the frameworks and checklists.
\end{abstract}

\section{KEYWORDS}

Instructional Quality, MOOC Quality, MOOC Quality Framework, MOOC Quality Checklist, Open Online Courses, Start@unito

\section{INTRODUCTION}

MOOCs, Massive Open Online Courses, can be defined by the following statement: "an online course designed for a large number of participants that can be accessed by anyone anywhere, as long as they have an internet connection, is open to everyone without entry qualifications and offers a full/complete course experience online for free" (Jansen et al., 2015). According to this definition, MOOC can potentially spread higher education and enhance the quality of life for millions of people. However, the basin of attraction of MOOCs contains mainly well-educated learners with a background in higher education and generally in possession of a qualification (Macleod et al. 2015). Another typical MOOC user is a learner already in employment. Moreover, by the definition stated above, it is not clear what is the threshold for an open online course to receive the $\mathrm{M}$ of "Massive", that is why in this work we will sometimes refer just to Open Online Courses (OOCs).

In the global education market, there are few platforms which contain thousands of MOOCs, created with the collaboration of universities and private companies. The growing trend of MOOCs raises issues about quality assurance. The first concern about quality regards the contents: MOOCs are courses consisting of different kind of educational resources, assessments, sometimes tutoring, usually peer-to-peer, rarely provided by academics. Higher education institutions usually outsource these components to third parties, for example, video recording of lectures, automatic grading programs, authentication services. The second concern about quality regards partnerships: for-profit education companies, educational publishers, global testing services collaborate with universities to provide MOOC, and this happens without internal academic capabilities. Thus, the focus on quality assurance is not only related to educational institutions. However, in (Ossiannilsson et al. 2015) note that partner organizations cannot easily be submitted to national higher 
education quality assurance standards and other regulatory instruments, since they were not designed to regulate such entities.

Apart from the ones listed above, MOOCs can serve different purposes. The main objective of the "Start@unito" project at University of Torino (Bruschi et al., 2018; Marchisio et al., 2019a), financed by Compagnia di San Paolo, is to improve the success rate of first year university students through the creation and dissemination of 50 OOCs available at https://start.unito.it, since a successful transition from secondary to higher education is a crucial challenge for both students and institutions (Barana et al., 2017). The project faced the major concerns about quality assurance. Since the project involves different kind of areas (sciences, humanities, economics, law, languages), there is a large difference in the typologies of contents and assessments according to the different need of the disciplines. Moreover, different kind of services collaborate to the various functionalities of the online platform: a Learning Management System (LMS), a Single-Sign On (SSO), an on-demand video service, an integrated Advanced Computing Environment (ACE) and integrated Automatic Assessment System (AAS).

This paper discusses issues related to the quality assurance of the OOCs provided by the project (not by single $\mathrm{OOC}$ ), according to frameworks provided by the large literature.

\section{STATE OF THE ART}

In this section we will analyze the main aspects that emerge in the literature related to the quality assurance of MOOCs. First, we will focus on the importance of MOOC, while, secondly, we will analyze quality indicators and possible frameworks.

\subsection{Importance of MOOC Quality}

MOOCs are more subjected to external scrutiny than campus-based higher education, which usually receive internal or students' evaluation. This can affect the national higher education system. In the UK, the Quality Assurance Agency (QAA) stated that MOOC providers should "ensure that they reflect the established reputation of UK higher education" (QAA 2014). Therefore, it is highly probable that the quality of MOOCs will be influenced by the support of national governments, too. More in general, assuring the quality of MOOCs is a responsibility of MOOC providers, cross-institutional partnerships and institutions, with guidance from national quality agencies.

MOOCs have an important relation with both online and open education. Openness does not mean only "free". In general, open education should remove barriers (Bates 2015). Thus, quality indicators should consider accessibility and usability issues.

When choosing between different MOOCs, the learner should be assured of their quality, by means of reviews and recommendations of other learners. Despite the large numbers of MOOC users, there is no prominent MOOC rating website. The only indicators for the learner are given by the brand reputation or the originating institution, sometimes the course author. However, (Daniel, 2012) remark that university brand is a poor measure of quality in online teaching, because reputations of academic institutions derives from research than teaching.

\subsection{MOOC Quality Indicators}

The concept of quality in online education is quite complex. According to (Jansen et al., 2017), the quality of MOOCs can be considered from the following four perspectives.

1. Quality from the learner's point of view. Different kind of learners, with different motivations and different backgrounds, interact with a MOOCs. Thus, while designing MOOCs, goals, expectations, learning behaviors and abilities should be considered to facilitate learning.

2. Quality connected to the pedagogical framework of the MOOC. MOOCs should be scale to an unlimited number of learners, with the principle that the teaching effort must not increase proportionally. Researches examine the choice of pedagogical model according to qualitative indicators for dialogue and interaction. 
3. Quality related to the input elements. The key role is played by instructional design (Margaryan et al., 2015). For example, (Costello et al., 2016) found flaws when analyzing multiple choice questions of several MOOCs.

4. Quality based on outcome measures. Data are going to play their part in this perspective. Possible indicators may include the number of learners completing a MOOC or achieving certification. However, not all learners want to follow till the end the path of a MOOC. Thus, completion rate has therefore been criticized (Aheran, 2018) when used to measure quality. Low values of generally used measures, such as retention and completion, may not indicate poor quality.

The quality assurance process involves different stakeholders. The first check is internally provided by the institution that is going to deliver the MOOC. The second step is provided by peer review, a person with experience in the topic and/or in online education. The third step is in the hands of the users, that should be entitled to leave feedback to the MOOC provider.

\subsection{Frameworks and Checklists}

Frameworks provide a useful tool for all the stakeholders involved in MOOC development. The QRF, Quality Reference Framework (Stracke et al., 2018), has been developed by the European Alliance for the Quality of Massive Open Online Courses (MOOCs), called MOOQ. It is a framework that analyze the needs and demands for MOOCs, in order to design, develop and implement new MOOCs and to evaluate and improve existing MOOCs. The framework is composed by 3 dimensions:

- Phases: Analysis, Design, Implementation, Realization, Evaluation.

- Perspectives: Pedagogical, Technological, Strategic.

- Roles: Designer, Facilitator, Provider.

Evaluation is a phase that surrounds all the other phases, it is the common background of every action in MOOC development.

National or international quality framework such as the OpenupEd Quality Label aims to supports institutions in the enhancement of MOOC quality, focusing on Quality Assurance processes in place (OpenupEd). OpenupEd developed some checklists that supports in self-assessing their MOOC development. One of these checklists concerns the quality of the design of MOOCs.

\section{RESEARCH QUESTIONS}

This research sourced as a necessity in order to understand the state of quality of the 50 OOCs provided in the framework of the start@unito project. The Research Questions (RQ) can be stated according to the following list items:

RQ1.How many are the quality indicators mentioned in the literature expressed in start@unito OOCs?

RQ2. What is the evaluation according to the OpenupEd checklist?

RQ3.How can be the evaluation phase improved according to the QRF with the focus on the Evaluation phase?

The three Research Questions highlight three main aspect that concerns MOOC quality. The individuation of general key aspects (RQ1), the need of a checklist to be used by universities designer and provider (RQ2), the meta-evaluation, i.e. the evaluation of the evaluation phase (RQ3).

The paper is not only devoted to the analysis of the quality of start@unito OOCs, but it will focus on a critical reflection around the various issues and topics connected to the RQs and the quality of MOOCs.

\section{METHODOLOGY}

This research, according to its questions, is divided into three parts. The first part addresses the presence of quality indicators (learners' point of view, pedagogy, input elements, outcome measures) inside the Start@unito experience, with reference to precise aspects of the project. The second part provides an evaluation of the quality of the OOCs according to a simplified version of the OpenupEd quality checklist. 
The entries of the marking scheme are: Target group, Overall goal, Learning objectives, Learning activities, Feedback mechanism, Study-time, Workload, Assessment. These entries are graded according to the 4-point scale:

1. NA (Not achieved),

2. PA (Partially achieved),

3. LA (Largely achieved),

4. FA (Fully achieved).

The third part provides a reflection on the adopted evaluation, considering some main points of the QRF framework. The analysis regards the 50 courses overall because it considers transversal solutions and objectives. It is not part of this work, but with the same scheme it is also possible to make a single course analysis, which could be useful for teachers who intend to prepare online courses.

\section{DISCUSSION}

In this section, beyond the general reflection on quality of MOOCs, we will focus on the specific validation and critical analysis of the OOCs provided by the start@unito project, following the list provided by the research questions.

\subsection{Start @ unito OOCs: Quality Indicators}

The first indicator is the learners' point of view. The main users that enters start@ unito courses are high school students, or first year students. There is a percentage of teachers that logged in, since we carried out experimentations (Marchisio et al., 2019c), but we do not know the exact number of the different extractions, since the data required to access the OOCs are simply First name, Last name and Email. Further data are only required when the user wants to obtain the certification.

The second indicator is pedagogy. All the university professors and postgraduates involved attended a training course (Marchisio et al., 2020) in order to develop skills about the main aspects related to the development of OOCs. The topic of the first training session was exactly about pedagogy connected to Open Online Education and about the concept of learning object. The participation to the meetings was not mandatory, thus we cannot ensure that all professors were committed to the pedagogical criteria.

The third indicator concern input elements. The other training sessions of the course (Marchisio et al., 2020) faced other aspects, like the technological and the organizational point of view, since the platform allows very different kind of contents (videos, documents, assessment, external resources, etc.) that the teacher need to master. Besides all the training, many contents show instructional problems, since not all the teachers followed the exact recommendations. This is not to be intended as a critical problem, since many teachers were approaching online education for the first time.

The fourth indicators are outcome measures. As evidenced in Section 2, it is very difficult to find which measure to adopt, since the "standard ones" are not considered to be relevant. In the case of the start@ unito OOCs it is even more difficult, since the project is quite young and more than half OOCs were opened in 2019, few of them at the beginning of 2020. Moreover, since the courses correspond to university module that, according to the different university courses, take pace in the first or in the second semester, the chance for student to sit the exam, which is compulsory to obtain the ECTS, may not have occurred. In our opinion, one year is the minimum amount of time in order to consider at least partially relevant the outcome measures. In fact, the data arising from the first 20 courses, completed in 2018, are very promising: even with this consideration and counting the newly opened courses, the average subscriptions to the courses is around 433 students and more than one thousand students already passed the exam, which is around the 5\% of all enrollments. The examinations of the last two months, January and February 2020, whose records still have to be closed, are missing: this could bring to an increase in the percentage of completion between 10-15\%, which is in line with the MOOC completion rates (Margaryan et al., 2015). There are of course students that uses the online contents, but do not take the examination. 


\subsection{Start@unito OOCs: Evaluation}

We are going to follow the checklist provided by OpenupEd about "Quality of the design of MOOC". The median and average of the results arising from the various categories is LA, as depicted in Table 1 below.

\subsubsection{Target Group}

Start@unito OOCs are accessible to all people, but users of the various target groups, the needs, the challenges and prior knowledge cannot be identified in the description of the course. The target group of university students is supported by different references according to their study program, but there is no other differentiation for other kind of users (teachers, professionals, etc.). The overall judgment for this category is LA.

\subsubsection{Overall Goal}

The overall objective of start@unito OOCs is described in a short video, duration around 4 minutes, introducing the main topics and themes touched by the course program. Moreover, even if not necessary for learning purposes, the video shows the professors that created the course, providing the reference to "a person behind the machine". Usually but not in every course, short sentences provide a little more insight about course contents. The overall judgment for this category is FA.

\subsubsection{Learning Objectives}

Every course describes learning objectives, but usually there is no statement about learning outcomes, nor in the main description neither in the single learning objects. There is reasoned coherence between course contents, teaching strategy and assessment methods, but the prior knowledge of each learning objective is rarely described. The overall judgment for this category is PA.

\subsubsection{Learning Activities}

The online activities allow participants to construct their own learning, but few activities ask students to communicate their results to others or to the system. The activities contain just one level of difficulty and one learning pathway, while complexity is increasing together with the knowledge of students. Various activities are proposed with different formats, but there is no peer-to peer interaction, forum or video conferences with tutors. The courses contain enough interactivity learner-to-content. The overall judgment for this category is PA.

\subsubsection{Feedback Mechanism}

The only feedback provided from start@unito OOCs is the automatic feedback, there is no academic tutor and no monitoring, apart from the evaluation process. Despite this, the course provides learners with regular feedback through self-assessment activities and tests, the number of feedbacks can be improved. The overall judgment for this category is PA.

\subsubsection{Study-time}

The students learn autonomously and self-paced, so there is no timing, no periodic live session. The total study time of all learning activities is scaled on the number of ECTS, that lies for start@ unito OOCs between 6 and 12 ECTS (1 ECTS equal around 25-30 hours of study). There was a lot of reflection about this topic in the design phase, it is a theme that professors care, thus the overall judgment for this category is FA.

\subsubsection{Workload}

The schedule of the course is such that the workload is suited for learners from the main target group. The start@unito OOCs are realistic in their pacing for the participant, accommodating to the personal rhythm, since the contents are always available, 24/7. The overall judgment for this category is FA.

\subsubsection{Assessment}

Some OOCs present a balance between formative and summative assessment. Automatic Formative assessment is essential for self-paced online courses. There are no measures appropriate to counter 
impersonation and plagiarism, however after completion students receive a certificate of completed learning activities that allows them to take the in-person exam at the university, thus the ECTS are obtained in a safe, reliable and valid way. The start@unito OOCs track score and progression. The overall judgment for this category is LA.

Table 1. Summary of the evaluation according to the OpenupEd checklist about "Quality of the design of MOOC" according to the scale: NA (Not achieved), PA (Partially achieved), LA (Largely achieved), FA (Fully achieved)

\begin{tabular}{llllll}
\hline Category & Judgment & Value & Category & Judgment & Value \\
\hline Target group & LA & 3 & Feedback mechanism & PA & 2 \\
Overall goal & FA & 4 & Study time & FA & 4 \\
Learning objectives & PA & 2 & Workload & FA & 4 \\
Learning activities & PA & 2 & Assessment & LA & 3 \\
\hline Median & LA & $\mathbf{3}$ & Average & LA & $\mathbf{3}$ \\
\hline
\end{tabular}

\subsection{Start@unito OOCs: Meta-Evaluation}

The QRF (Stracke et al., 2018) focus on key Quality Criteria and Checklist for analyzing, designing, implementing, realizing and evaluating a MOOC. The quality criteria and checklist defined below are action items for activities in the evaluation phase. The QRF Quality Checklist asks questions intended for both novices and experts in MOOC design and development.

\subsubsection{Evaluation Planning}

In the design of the evaluation phase, the criteria or evaluation objectives must be set. In the case of the start@ unito OOCs the measurable is given by improving the outcomes of the evaluation criteria of first year university students, which is given by the number of ECTS obtained, with many possible evaluation focus on learners engagement, with data mainly provided by their interaction with the online platform. The evaluation process still must start, and it will take place periodically, possibly once a year. A general overview of the trend is reported at the meetings of the Scientific Committee of the project. As a general approach, the design phase and the realization phase were evaluated.

\subsubsection{Evaluation Realization}

Among the various tools, we adopted questionnaires in order to gather precious feedback from students and high school teachers in order to assess the realization phase and from university professors in order to assess the design phase. Moreover, a first approach with learning analytics tools provided feedback on all learner activities (Marchisio et al., 2019b).

\subsubsection{Evaluation Review}

There is no precise protocol about data review, but all interested core stakeholders represented in the MOOC design team take part in the analysis and discussion of the results. The Scientific Committee concern about the overall progress, while the single university professor concern about its own specific OOC. The documentation about the data is strictly confidential and internal to the people involved in the project management, while documentation of findings, from learning analytics and other forms of data are published as open research items. Data collected provided recommendations for improvement.

\subsubsection{Improvements and Optimization}

Technical administrators of the platform and project managers are active in the experimentation of new kind of analytics and frameworks. It will be useful to consult an evaluation team to oversee the possible implementation of the recommendations obtained from the evaluation review process, bug fixing and operational improvements. 


\section{CONCLUSION}

This research work provided an implementation of the analysis of MOOC quality with a marking scheme that evaluates 8 main points that addresses the quality of MOOCs according to studies and reflections provided by the rich literature. The points are marked with a 4-point scale. Moreover, a specific analysis in the local setting of the 50 start@unito OOCs at the University of Torino. Recalling the research questions, we can collect the ideas arose:

RQ1.Indicators are necessary as guidelines to MOOC designers and providers. Start@ unito started its experience with a training program of academic staff and professors, giving emphasis to some of the listed indicators. Even trainers were exploring new strategies and ideas during the training course. Some university professors were quite new in the world of online education. The results from this point of view are different between courses, but even the ones in which the quality is lower are encouraging since academics have been positively engaged and admitted in a different way of teaching.

RQ2.The team of OpenupEd created useful checklists, which allows any MOOC designer and provider to assess himself. The reference table about quality seems to promote the start@unito OOCs. Not all the categories obtained a good mark according to the gridlines and the marking scheme over a 4-point scale, but the average between the various aspects is positive, and states that the quality objectives are largely achieved.

RQ3. Some of the criteria for a good evaluation phase are respected, since there is a literature of publications with results obtained from data arising from students, teachers, university professors and other users. It is needed more planning and review of results from all stakeholders, that may not be aware of the actual status of learner activity. Learning analytics are a promising way to go to explore new patterns in online learning processes.

Self-reflection on the quality of an experience provides an important insight and feedback. In this case it is even more important since there were many different actors involved in the design of the online courses. The marking scheme is quite basic in other to give a global overview on the quality of a MOOC, but it can be furtherly refined, with other entries or sub-entries. As a global view of the results and of the answers to the three research questions, start@unito appear as an important experience in the online education panorama. start@unito will continue to spread education for many years, trying to help students in their transition between secondary and higher education. Other evaluations could give more interesting results according to the maturity of the experience. As an example, future work will involve data analysis on user's activity in the online platform, students marks from the self-assessment system, outcomes arising from the exam board and the correlation between these data, in order to understand the usefulness of this experience from different point of views (students, teachers, professors, university).

\section{REFERENCES}

Aheran, A., 2018. Stop Asking About Completion Rates: Better Questions to Ask About MOOCs in 2019. Retrieved from https://www.edsurge.com/news/2018-11-28-stop-asking-about-completion-rates-better-questions-to-ask-aboutmoocs-in-2019

Barana, A., Bogino, A., Fioravera, M., Marchisio, M., Rabellino, S., 2017. Open platform of self-paced MOOCs for the continual improvement of academic guidance and knowledge strengthening in tertiary education. Journal of e-Learning and Knowledge Society, 13(3), pp. 109-119. https://doi.org/10.20368/1971-8829/1383

Bates, A. W., 2015. Teaching in a digital age. Guidelines for designing teaching and learning for a digital age. Ed. Tony Bates Associates Ltd. Retrieved from http://opentextbc.ca/teachinginadigitalage/, Last accessed March, 3rd 2020.

Bruschi, B., Cantino, V., Cavallo Perin, R., Culasso, F., Giors, B., Marchisio, M., Marello, C., Milani, M., Operti, L., Parola, A., Rabellino, S., Sacchet, M., Scomparin, L., 2018. Start@unito: a Supporting Model for High School Students Enrolling to University. IADIS International Conference Cognition and Exploratory Learning in Digital Age 2018, pp. 307-312.

Costello, E., Brown, M., and Holland, J., 2016. What Questions are MOOCs Asking? - An Evidence-Based Investigation. Proceedings of the European Stakeholder Summit on experiences and best practices in and around MOOCs, pp. 211-221. 
Daniel, J., 2012. Making Sense of MOOCs: Musings in a Maze of Myth, Paradox and Possibility. Journal of Interactive Media in Education, 2012(3), p.Art. 18. http://doi.org/10.5334/2012-18

Jansen, D., Rosewell, J., and Kear, K., 2017. Quality Frameworks for MOOCs. In: Jemni M., Kinshuk, Khribi M. (eds) Open Education: from OERs to MOOCs. Lecture Notes in Educational Technology. Springer, Berlin, Heidelberg. https://doi.org/10.1007/978-3-662-52925-6_14

Jansen, D., Schuwer, R., Teixeira, A., and Aydin, C. H., 2015. Comparing MOOC Adoption Strategies in Europe: Results from the HOME Project Survey. The International Review of Research in Open and Distributed Learning, 16(6). https://doi.org/10.19173/irrodl.v16i6.2154

Macleod, H., Haywood, J., Woodgate, A., and Alkhatnai, M., 2015. Emerging patterns in MOOCs: Learners, course design and directions. TechTrends, 59(1), pp. 56-63. http://dx.doi.org/10.1007/s11528-014-0821-y

Marchisio, M., Operti, L., Rabellino, S. and Sacchet, M., 2019. Start@ unito: Open Online Courses for Improving Access and for Enhancing Success in Higher Education. In Proceedings of the 11th International Conference on Computer Supported Education - Volume 1: CSEDU, ISBN 978-989-758-367-4, pp. 639-646. http://doi.org/10.5220/0007732006390646

Marchisio, M., Rabellino, S., Roman, F., Sacchet, M., and Salusso, D., 2019b. Boosting up Data Collection and Analysis to Learning Analytics in Open Online Contexts: an assessment Methodology. Journal of E-Learning and Knowledge Society, 15(3), pp. 49-59. https://doi.org/10.20368/1971-8829/1135048

Marchisio, M., Rabellino, S., Sacchet, M., and Salusso, D., 2020. From desk to desktop: the integration between classroom and online teaching from the teachers' perspective. Reports on E-Learning, Media and Education Meetings, 8(1), 43-48. Retrieved from https://www.je-lks.org/ojs/index.php/R-EMEM/article/view/1135104

Marchisio, M., Sacchet, M., and Salusso, D., 2019c. Instructional design to "train the trainers": The start@ unito project at the university of turin ", Multi Conference on Computer Science and Information Systems, MCCSIS 2019 Proceedings of the International Conference on e-Learning 2019, pp. 195-202, 2019. https://doi.org/10.33965/el2019_201909F025

Margaryan, A, Bianco, M., and Littlejohn, A., 2015. Instructional quality of massive open online courses (MOOCs). Computers \& Education 80, pp 77-83. http://dx.doi.org/10.1016/j.compedu.2014.08.005

OpenupEd. Retrieved from https://www.openuped.eu/, Last accessed March, 3rd 2020.

Ossiannilsson, E., Williams, K., Camilleri, A. F., and Brown, M. L., 2015. Quality models in online and open education around the globe: State of the art and recommendations. Retrieved from https://www.pedocs.de/volltexte/2015/10879/pdf/Ossiannilsson_et_al_2015_Qualitymodels.pdf, Last accessed March, 3rd 2020.

QAA. (2014). Statement on massive open online courses. Retrieved from https://dera.ioe.ac.uk/19652/1/QAA-positionstatement-MOOCs.pdf, Last accessed March, 3rd 2020.

Stracke, C. M., Tan, E., Texeira, A., Pinto, M., Vassiliadis, B., Kameas, A., Sgouropoulou, C., and Vidal, G., 2018. Quality Reference Framework (QRF) for the Quality of Massive Open Online Courses (MOOCs). Online available at www.mooc-quality.eu/QRF, Last accessed March, 3rd 2020. 